\title{
Voluntary Switching of Visual Motion Rivalry Estimate Human Heart Rate
}

\author{
Ahmad Yousef ${ }^{1}$ \\ ${ }^{1}$ School of Computational Science and Engineering, McMaster University, Hamilton, Ontario, Canada \\ *Correspondence: mohamas2@mcmaster.ca
}

\begin{abstract}
We showed that deep breathing and voluntary hand movements are able to effectively and timely alter visual bistable perception, see reference 1 and 2 . Deep breathing and voluntary hand movements require cognitive control, however, deep breathing causes stable respiratory sinus arrythmia (RSA). We decided to achieve this study because we have previously claimed that the RSA process is the governor of the visual motion rivalry through the deep breathing, see reference 1. We therefore suspect whether every kind of volitional switching to visual motion rivalry has correlation to the heart rate. Expectedly, we found that deep inhalation which is associated with the perception of the actual visual martial is able to increase the heart rate; and deep expiration which is correlated to the perception of motion reversals
\end{abstract} is able to decrease the heart rate. Astoundingly, for the voluntary switching through the hand movements, we found that when the human subjects move a pen in harmony with the actual physical direction which results in the perception of the original materials of the visual stimulus; the heart rate is increased. Illusory motion reversals, which appears when the pen is moved in the opposite direction of the actual motion, are correlated to heart rate deceleration!

\section{Introduction}

Respiratory sinus arrhythmia is an accommodation process which occurs during breathing cycles; namely the heart rate accelerates for the inspiration and decreases for expiration, see references 3 , and 4. Needless to mention, the dorsolateral

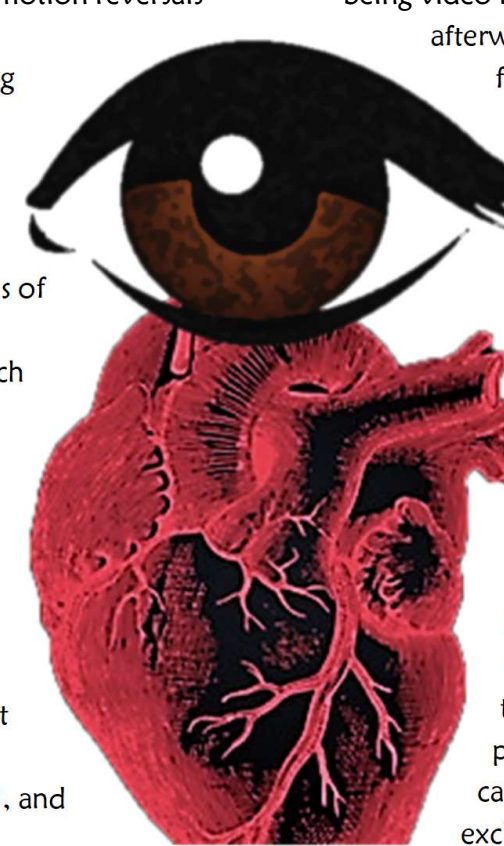
prefrontal cortex has important role in the executive functions (that shall include decision for deep breathing as well), see reference 5. It's the highest cortical area which is responsible for motor planning, organization, and regulation. It is not only responsible for decision making but for motor signaling, which is neurologically connected to the premotor cortex and subsequently the motor cortex. Cognitive action is therefore a sophisticated neurological task; it starts from the prefrontal cortex towards the primary motor cortex, to the muscles' nerving ends through the spinal cord that eventually yield body movements. The DLPFC also could signal the primary olfactory cortex to eventually control the breathing process. Because of the phenomenal effect of respiratory sinus arrythmia on the visual motion rivalry as well as the heart; we decided to deeply study whether cognitive control of the visual motion rivalry have direct influence on the heart rate. Needless to mention, when the pupil is dilated, retinal peripheries but not retinal centralis tend to see illusory motion reversals, see references 6 , and 7 .

\section{Materials \& Methods}

Two human subjects were recruited to participate in this study. Polar $\mathrm{H} 10$ sensor had been used to collect the heart rate values with sampling rate of $200 \mathrm{~Hz}$, and the sensor runs at $(-41 \mathrm{~dB})$. The visual experiments are presented through non-touch screen with refresh rate of $60 \mathrm{~Hz}$. Throughout the experiments, the human subjects are being video recorded with $4 \mathrm{~K}$ camera, these videos are afterwards used to manually determine the following time intervals: harmonized and disharmonized voluntary hand movements, as well as deep inhalation and exhalation. Two experiments have to be tested; each for them has two sections, reference section, and deep breathing section / voluntary hand movements section, see reference 8 . In experiment 1 (deep breathing experiment), the stimulus subtends 12.9 DVA length and 1.37 DVA width and consisted of 9 cycles, and runs at constant speed of 12.9 DVA/sec. Each human subject has to experience the experiment for three times for data finetuning. In the beginning of experiment 1 , the protocol says that any human subject who cannot see illusory motion reversals must be excluded; however, since we had chosen the subjects with dilated pupils; both of them were able to see the illusory motion reversal from the first time. In the breathing section of experiment 1 , the human subjects have to follow certain protocol, namely, take a deep inhalation, execute a profound exhalation, take an extremely short break, and then repeat the cycle until the end of the trial. Subjects are not instructed to shorten or elongate their breathing cycles, we kept them to choose their own comfortable timing; however, we were strict about 'deep breathing'. Each human subject is requested to report the influence of inhalation or exhalation on the aforementioned stimulus and to compare it with the generic references. As expected, same results of reference 1 are reported. Experiment 2 is exactly similar to 
experiment 1; however, human subjects are requested to perform "voluntary hand movements" cycle. In another word, in each cycle, the human subject has to move the pen in harmonized way, i.e., with the motion's direction of original martial; and then in disharmonized way, i.e., against the motion's direction of the original material, for further details, see reference 2. Important to mention, we divided each breathing cycle includes to halves, the inhalation half cycle, and the expiration half cycle. The voluntary hand movements cycle is also divided into two halves, the harmonized movement is the first half cycle, and the disharmonized movement is the second half. In our primarily analysis of the present of the cardiological data, we noticed that it's not proper to have a baseline for the heart rate, not only because the baseline should be dynamic, but because of the clear visibility of "peak values" whether these values are positive or negative. We also notice that the heart rate variability metric is concerned about the variability not the positivity and the negativity around a dynamical baseline that are produced during the aforementioned cognitive control processes. We therefore conceptualize the following metric: Peak-toAverage-Heartrate-Ratio (PAHR); a metric that shall give a holistic value of whether the heart accelerates or decelerates in each of the aforementioned cognitive processes (deep inhalation, deep expiration, harmonized hand movement, and disharmonized hand movement; the metric should be given as follow:

PAHR $=\frac{\sum_{n=1}^{n=m} \frac{(\text { Peak HR })_{\text {half cycle }}}{(\text { Avg.HR })_{\text {full cycle }}}}{m}$

Where $n$ indexes the number of a specific cycle, $m$ is the overall number of cycles; peak HR is the greatest BPM value in each corresponding half cycle "each half cycle temporarily represents the time interval of one of the aforementioned cognitive processes per each unit cycle"; and finally, the $\mathrm{Avg}$. $H R$ represents the average value of the entire unit cycle. To illustrate the application of equation (1) on a single breathing cycle (two halves); we use an excerpt of the collected physiological data (see below), i.e., a unit cycle from the breathing experiment:

PAHR $_{\text {inhalation }}=\frac{76.1 \mathrm{bpm}}{73.4 \mathrm{bpm}}=1.0368$

PAHR $_{\text {expiration }}=\frac{71.8 \mathrm{bpm}}{73.4 \mathrm{bpm}}=0.978$

Equations 2 and 3 clearly state that if the PAHR value is above 1 then the heart rate is accelerated, and the peak has a positive value, but if the PHAR value is below 1 then the heart is decelerated, and the peak is negative.

Data Collection: Behavioral and Cardiological To comply to the declaration of Helsinki, however, we confirmed comfortable settings for each subject. The behavioral data were collected manually as mentioned in the previous section. The first cycle was omitted from the cardiological analysis to ensure the stability. For all of the experimental sessions; all of the cardiological data are analyzed and PAHR is calculated for each session; and then all of the PAHRs from all of the sessions that includes the data from the two human subjects are then averaged, and the standard error of the mean is evaluated. The net result of the aforementioned mathematical processes are as follow:

PAHR $_{\text {inhalation }}=1.0394 \pm 0.0124$

PAHR $_{\text {expiration }}=0.963 \pm 0.0043$

$\mathrm{PAHR}_{\text {harmonized move }}=1.0211 \pm 0.0027$

PAHR $_{\text {disharmonized move }}=0.974 \pm 0.0013$

\section{Results \& Discussion}

Clearly, the PAHR for inhalation and harmonized hand movements is above 1, namely, it shows an increased heart rate with positive peaks; however, for expiration and disharmonized hand movements, the PAHR is below 1 , namely, decreased heart rate with negative peaks. Interestingly, deep breathing has greater heart rate variability than voluntary hand movements.

\section{Essential Notifications}

Readers should have direct access to the stimuli, see reference 8 . Advisably, download the videos for the best quality. Be sure that you have dilated pupils before the experiments, if you do not see illusory motion reversals in the first session, then you are still not ready. In order to dilate the pupil, watch the video in fifty percent brightness, and have some caffeine intake, see reference 9. Needless to say, there are several articles talk about the interactions between the heart and the perception, see reference 10; however, none of them had studied the link between the effect of manual switching perceptual rivalry on the heart.

\section{Transactional References}

[1] Yousef, A. 2019. "Deep Breathing Alters Visual Motion Perception." PsyArXiv. doi:10.31234/osf.io/up3sa.

[2] Yousef, Ahmad. 2020. "Voluntary Movements Discipline Audiovisual Perception." PsyArXiv. doi:10.31234/osf.io/bqr6u.

[3] Berntson etal., (1993). Respiratory sinus arrhythmia: autonomic origins, physiological mechanisms, and psychophysiological implications.

Psychophysiology.

[4] C. Ludwig, (1847). On the influence of respiratory movements on blood flow in the aortic system. Leipzig.

[5] Goldman-Rakic, etal. 1995. "Architecture of the Prefrontal Cortex and the Central Executive". Annals of NewYork Academy of Sciences.

[6] Yousef, A. 2019. "Spatial Attention Dilates Time and Length." PsyArXiv. doi:10.31234/osf.io/qazj9.

[7] Yousef, A. 2019. "Retinal Peripheries Generate Illusory Motion

Reversals." PsyArXiv. doi:10.31234/osf.io/gfmpe.

[8] You may find the stimuli in the following link:

https://drive.google.com/drive/folders/1kPoiOkY8a-B4-

x2MLA7FMQcGclUZSJ5o

[9] Abokyi, etal.,(2017). Caffeine intake is associated with pupil dilation and enhanced accommodation. Eye (Lond) Journal.

[10] Yousef, A. 2019. "Rescuing Lives: When Cardiology Interweaves with Cognitive Neuroscience." PsyArXiv. doi:10.31234/osf.io/fjmy9. 